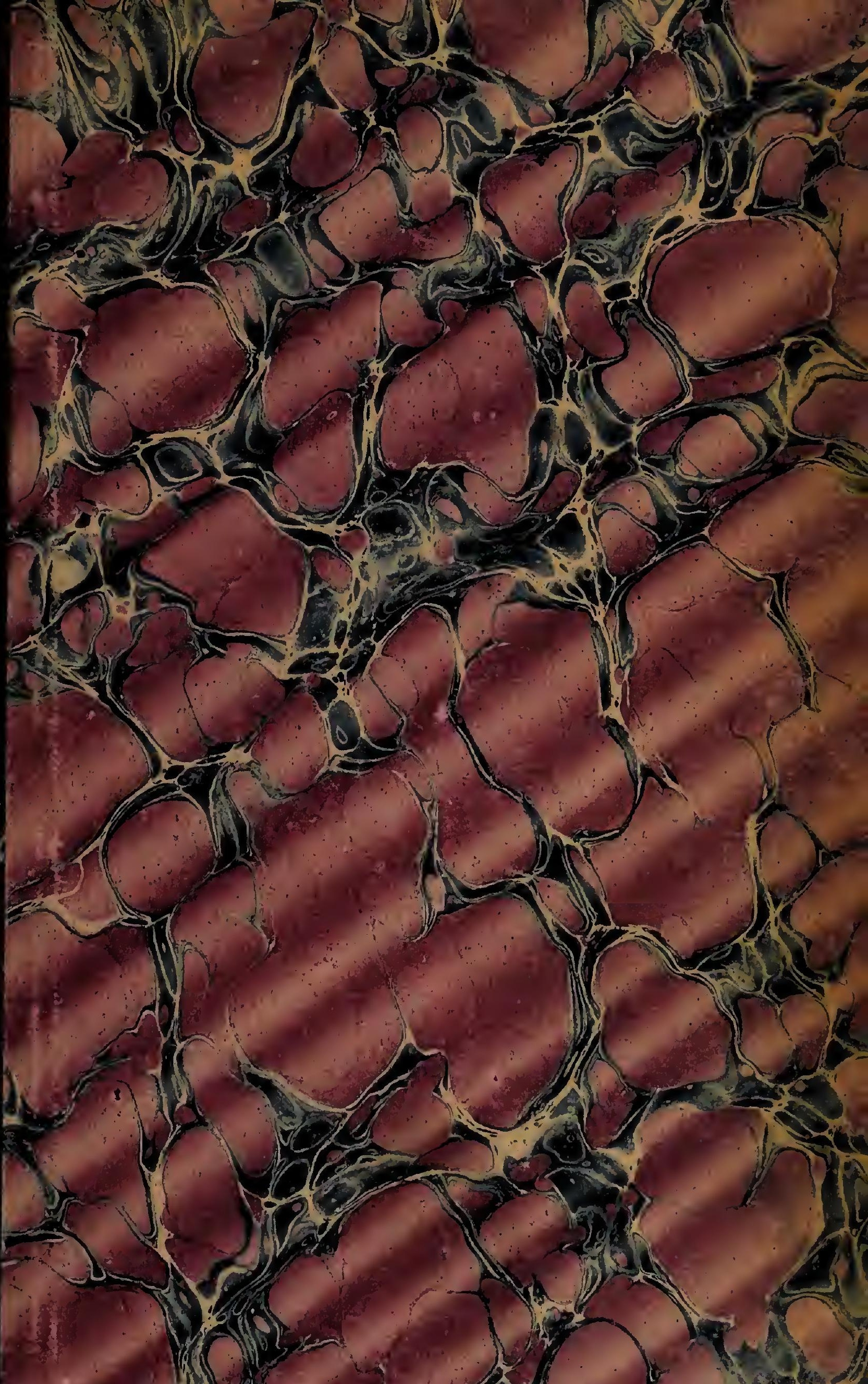




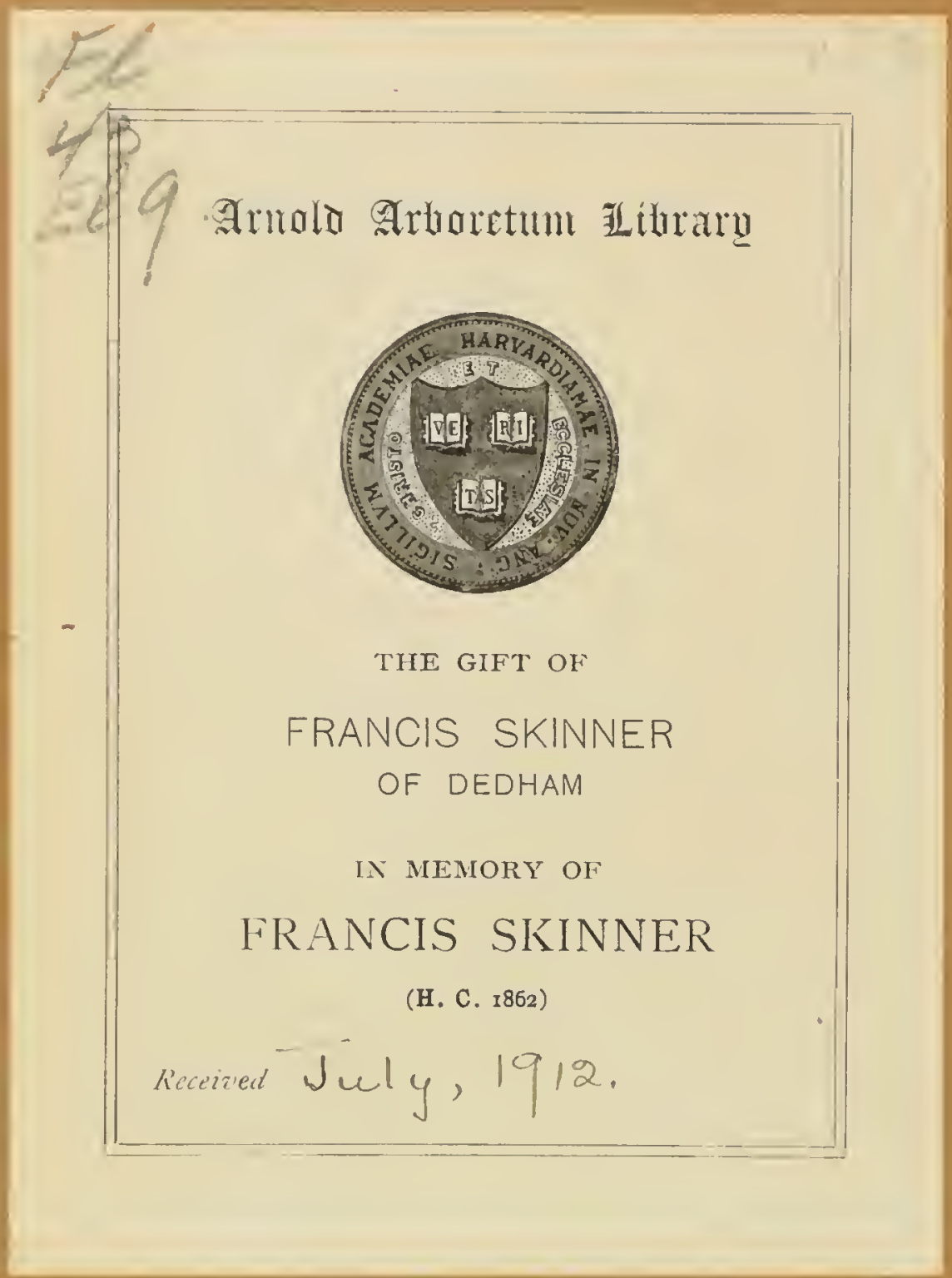






\section{FASCICVLVS PLANTARVM}

E

FLORA MARGGRAVIATVS BARVTHINI

DISSERTATIO INAVGVRALIS MEDICA

$$
\text { Q V.AM }
$$

SVMMI NVMINIS AVSPICIO

ACADEMIAE FRIDERICO ALEXANDRINAE

RECTORE MAGNIFICENTISSIMO

SERENISSIMO PRINCIPE AC DÓMINO

\section{O M I N O}

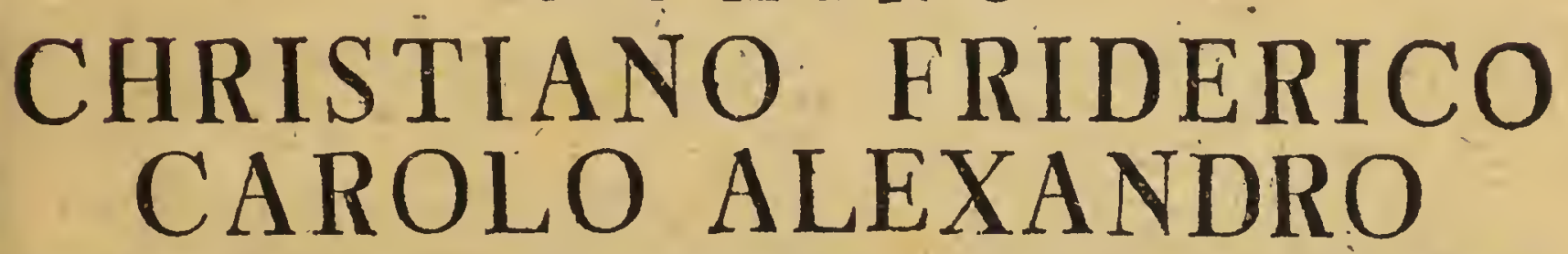

MARGGRAVIO BRANDENBVRGICO BORVSSIAE SILESIAEQVE DVCE BVRGGRAVIO NORLMBERGENSI VTRIVSQVE PRINCIPATVS REL; REL.

EX DECRETO

GRATIOSI. MEDICORVM ORDINIS

\section{PRO GRADV DOCTORIS}

OBTINENDO

DIE SEPT. MDCCLXXXVI.

DEFENDET

IOANN. CASPAR. PHILIPP. ELWERT SPIRENSIS

INSTITVTI MORVM ET ELEGANT. LITTERAR. ERIANGENS. SODALIS.

E R L A N G A

TYPIS KVNSTMANNIANIS. 
Ning12:2
26810 


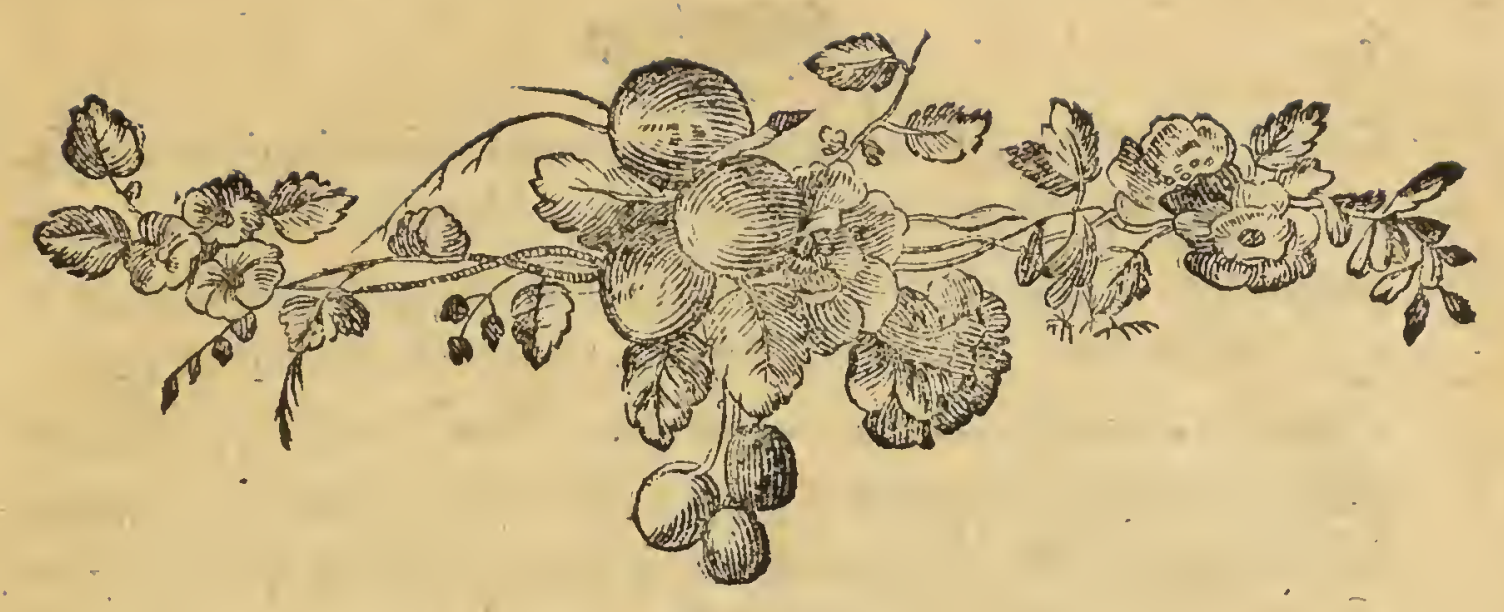

\section{P.R A EFATIO.}

um a prima inde iuventute tempus ab aliis negotiis liberum in cognitione naturae, eiusque varialum rcrum-perfcrutatione, collocarem, tum colles potius eligebam camposquc patentes, quam claufae cellae angultias, ubi induftria haec mea et oblectatio verfaretur. Praeci. pua vero cura mea in rei herbariae cognitione, varietate quippe fua et ad oblectandum animum iucundiffima, et ad ufis futuri medici uberrima, occupabatur. Hoc eodem confilio non folum diligentiflime interfui excurfioribus botanicis, quas rei botanicae difcipulorumque gra. tia et commodo ftatis diebus inftituere folet Vir mea laude maior et junicuique doetrinae huius perito notiffimus, III. SCHREBER, praeceptor et fautor meus pia mente femper venerandus; verum etian jpfe ut vicinum 
Erlangae agrum, fic etiam remotiores Baruthini principatus regiones identidem peragravi. Itaque diverfis anni temporibus agrum quoque perquifivi Muggendorfiantm, qui non admodum forfitan diffimilis quibusdam Helvetiae regionibus, variisque montibus iam fatis acclivis, Jargam praebet multarum diverfique generis plantarum copiam inveftiganti. In quo indagando, cum colleetis perpluribus plantis, quae alibi rariores funt inventu, bene pofitam effe operam cognoviffem, eo maiori incenfus fum cupiditate, fuperiores eosque altiores mantes et alpes quafi terrae Baruthinae, celebre illud iugum Piniferi adeundi. In quo itinere optima fane fortuna mea contigit ut comitem me adjungere licuerit acuto ifti rerum naturae fcrucatori, itinere in American fufcepto, multisque ibi deteetis plantis claro, celeberrimo schoeprio, medico aulico Baruthino, cuius in me benevolentiam, promtamque benignitatem nunquam dignis fatis laudibus celebrare potero. Inde redeunti mihi in agro quoque Baruthino per tempus quoddam verfari licuit, ubi fruteta faxorum et rupium circa ea potifimum loca, quae Eremitage, Fantaifie, Sanspareil, montisque Sopbiae nominibus celebrantur, perreptans largam variarum plantarum meffem reportavi. Huius igitur itineris atque otii mei ftatui nunc, hac differtationis inauguralis fcribendae opportunitate oblata, rationem quafi aliquam reddere, et earundem plantarum, quas in variis hisce locis collegi, nomina ac defcriptionem quandam cum peritis et aequis rei botanicae arbitris communicare; magnum iftud quondam orbis botanici 
nici fidus, b. LINNEvm loquor, imitatus, qui variis difertationibus academicis catalogos plantarum, in regionibus modo maioris, modo minoris ambitus provenientium exhibuit. Ut vero fingulas plantas levifime et absque multis longorum noninum ambagibus indicare poffem, ciusdem immortalis Viri nomina generico-trivialia adhibui, eaque ad normam fyftematis digeffi; exceptis illarum ftirpium, quae in libris a. b. Viro evulgatis defiderantur, nominibus, utpote quae à aliis auctoribus mutuari oportuit.' In Lichenibus autem dividendis et nominandis Viri in horum cognitione verfatiflimi, cel. G. F. Ho F rMANI, mili amicillimi, auctoritatem potiorem habui. Loca, in quibus minus vulgares plantae habitant, tantum indicavi, ut et nomina, quae mihi innotuerunt, indigena, adiectis quibusdam botanicis occonomicis et medicis obfervationibus. In quibus, fi quando ufus plantarum quarundam medicus iam aliunde, vel ex ipfis differtationibus hoc loco ventilatis, fatis notus erat, hoc egi, ut vires earum falutares, de quibus iam conftabat, aut ambigebatur, novis experimentis confirmarem et illuftrarem. Cui rei maxime fane profuit, inftituto clinico, quod fub Ill. WENDTIO, praeceptore fumme venerando, in hac Academia floret, per aliquod tempus interfuife. Caeterum lectorum indulgentiae hos meos labores commendo, fpe fretus, non defuturos effe, qui hunc Catalogum etfi non totius. Florae Baruchinae, tamen complurium Specierum ad eam referendarum, benevole excepturi, meosque conatus aequi bonique confulturi fint.
A 3
I. MONAN- 


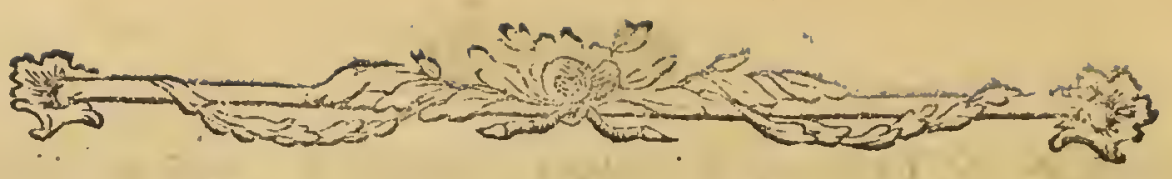

I. MONANDRIA. Digynia. Vtricularia vulgaris.

Callitriche verna. (circa Erlang.) - - autuminalis. (ibid.) - Verbena officinalis.

6. Stellaria fol, imis linearib. Lycopus europaeus. fupremis fubrctundig. HALL. Salvia-pratenfis. 553. LEERS fl. herb. p. r.

\section{DIANDRIA. Monogynia. Anthoxanthum odoratum.}

\section{Digynia.}

Liguftrum vulgare.

Circaea lutetiana.

Veronica $\int p i c a t a$.

- - officinalis.

- - Serpyllifolin.

- - Beccabunga.

- - Anngallis.

- - Scutellats.

- - Teucrium.

- - Clamaedrys,

- - - agreftis.

- $\therefore$ - arvenfis.

- - bedernefolia.

- - triphyllos.

- - verna.

Pinguicula vulgaris. (circa Pegniz, Baruth. ad mont. pinifer.)
'III. tr tandria. Monogynia. Valeriana dioica.

- - officinalis.

- - locifta olitoria.

Iris germanica.

- - fabirica (circa Tennenlohe.)

Schoenus compreffics.

- - albus (im Röthelweiher prope Defendorf.)

Cyperus Alaveficus.

- - fufcus.

Scirpus paluftris.

- - acicularis.

- - Setaceus.

- - lacuffris.

- - Syluaticus.

Erio-

Veronica agrefis. Caulis diffufus, teres, hirfutus. Folia calycis ovata, glabra, floralia alterna, fubovata incifa, pedunculi foliis longiores.'

Veronica arven/is. Caulis procumbens, hirfutus; rami plerique oppofiti, folia floralia alterna brevifime pedunculata, oblonga integerrima. Ca. lyx hirfutus. 
Eriophorum vag natum:

- - - polyftacbyon.

Nardus fricitn.

\section{Digynia.}

Phalaris arundinacea.

- - pbleoides.

- - oryzoides: (circa: Eil. )

Panicum Crus galli. a. B.

- - fanguinale. $\boldsymbol{\alpha}_{0}$ Bi.

Phleum pratenfe.

- - nodofim.

Alopecurus praten/is.

- - agreftis.

- - geniculntus.

Milium effufum.

Agroltis $\int$ pica venti.

- - arundinacea.

- canina.

$\longleftarrow$ - Jtolonifera:

- capillaris.

- alba.

Aira aquatica.

- cespitofa.

- fiexuofa.

- cancfiens.

- crryopbyller.

Melica nutans.

- uniflora RETz.

- caerulen.

Poa nquatica.

- trivialis.

- onguftifolia.
- pratenfis.

Poa anniua.

- Eragroftis. (Erlang.)

- compreffa.

- criftata.

Briza medin.

Dactylis glomernta.

Cynofurus criftatus.

- - - crevuleus.

Feftuca ovina.

- - rubra.

- - du-iufcula.

- - elatior..

- - decumbens.

- - fiuitans.

Bromus fecalinus.

-. - mollis.

- - giganteus.

- - - fterilis.

- - pinnatus.

- - gracilis WeIGel.

Avena elatior.

- fatua.

- pubéfiens.

- flavefiens.

Arundo Pbragmites.

- Calamagroftis.

Lolium perenne.

- $\quad-\quad$ temulentum:

Elymus caninus.

- - europaeusi(Muggendorf.)

Hordeum murinum.

Triticum repens: 


\section{Trigynia.}

Montiafontana.(Erl.Fichtelb.etc.)

Holofteum, umbellatum.

\section{TETRANDRIA. Monogyn.}

Globularia valg aris. (Muggend.)

\section{B. coroll. albis.}

Dipfacus fullonzm.

- - lacirintus.

- - pilofus (prope Erlang.)

Scabiofá fuccifa.

- - arvenfis.

Sherardia arvenfis.

Afpcrula odorata.

- - arven/is. (c. Muggend.)

- - cynancbica. (ibidem.)

Galium paluftes.

- uliginofuvt.

- Spurium. (Ratlisberg.)

- afperum inall. (Rathsb.)

- muftriacum JACQUiN. (ib.)

- vcrum.

- Mollugo.

- - Syluaticum.

- Aparine.

Plantago maior.

medin.

- lenceolata.

Centunculus minismus. (c. Bubeirr.) Sanguiforba officinalis:,

Cornus fanguizien.
Trapa ratans. (circa Weiffendorf.)

Alchemilla vulgaris.

B. hirfuta.

Digynia.

Aphanes arvenfis. (c. Ell. Baruth.) Cufcuea europaea.

- Epitbynum.

$$
\text { Tetragynia. }
$$

Potamogeton uatans.

- - perfoliatum.

- - $\operatorname{denfum}$.

- - lucens.

- - crifpum.

- - compreffum.

- - 'marinum. (c. Bayersd.)

- - gramineum.

- - prysilum. (Erl. Baruth.)

Sagina procumbens.

- erecta. (Erlang. Baruth.)

V. PENTANDria. Monogyn.

Myolotis frorpioides.

Var. arvers. paluffr. suiss.

- - Lappula.

Lithofpermum officinale: (Muggd.z

- - - avenfe.

Anchura officinalis. (Enlang:)

- - anguftifolia.

Cynogloffum officinale.

Globularia vulgaris folis omnibus ov tis obtufis integerrimis.

Galii ereri fuccus exprefi, in epilep . feltci fucceft: adhibitis en. 
Pulmonaria officinalis.

$$
\text { B. maculo/a. }
$$

Symphytum officinale.

Borago officinalis.

Lycopfis arvenfis.

Echium vulgare.

Primula veris. B. elatior.

- - farinofa. (ad Streitberg.)

Menyanthes trifoliata.

Hottonia paluftris. (c. Möhrend.)

Lyímachia vulgaris.

- - nemorum. (Rathsberg.)

- - mumularis.

Anagallis arven/is.

Var. coroll. phoen. caerul. ef lusea.

Convolvulus arvenfis:

- - - Sepium.

Campanula rotundifolia.

- - patula.

- - Rapunculus.

- - perficifolir.

-. - - rapunculoides.

- - Trachelium.

- - glonerata. (Erlang.)

- - Spcculum. (Tennenl.)

Phytevma Spicata. (Rathsberg.)
Lonicera Periclymenum. (Stręitb.)

- - Xyloftezm.

- nigra. (Baruth. Culmb.)

Verbafcum Thapfus.

- - Var coroll. alba.

- - Lyclonitis.

- - - nigumn.

- - Blattaria. (Bruk.).

Datura Stramonium.

Hyofcyamus niger.

Atropa Belladonna.(Rarsb.Sansp.)

Phyfalis Alkekengi. (Erl. Baruth.)

Solanum nigrum.

- Var. baccis buteis.

- - Dulcamara.

Chironia Centaurium.

Rhamnus catborticus. (Rathsb.)

- Frangula. (ibid.)

Evónymus europacus.

- - latifoizus. (Rathsb.)

Ribes rubrum. (prope Pegniz.)

- - alpinum. (Streitberg.) (nom. indig. Kizcnlbere.)

- nigrun. (Muggendorf.)

- uva crifpa.

Hẹdera Hclix.

The:

Atropa Belladonna externe in Emplaftro fecundum Difpenf. Brunsvicenf. parato, in glandulis induratis egregios effectus edidit. Spiritus e baccis Belladonnae per fermentationem praeparatus et ad uncias duas a viro robufto epotus, nullam, nifi folitam, fpiritus vini fim. plicis, vim exferuit.

Decoet. Dulcamaras in arthritide et fpafmis rhermaticis feliciter adhibitum'ent. 
Thefium lizop'yllum. (Muggend.) Caucalis grandifora. (Mugrend.) - alpinum. (Erlang.) - - laifoina. (Ratlsberg.)

Vinca miann.

Afclepias Vincetoxicum. (Bermek.) Herriaria glabra.

- birfita. (bei d. Dof. Brïke.).

Chenopodium bonus. Henricus.

$\therefore$ - - rubrum.

- - - murale.

- - - alhum.

- - - viridle.

- - bybridum.

- - - glaucum.

-.. - Vilvaria.

- - polyfpcrmum.

Vlmus campeftris. (Rathsberg:) - daucoides. (ibid.)

- leptop'sylla. (Baruth.)

Conitum maculatum. (Wolísfelden.) Selinum. Carvifol:a.

- - paluftre.

Athamanta Cervaria. (Tennenloh)

- - Libanotis. (Muggendorf.)

- - Oreofelinim..(Erl. Tennl.)

Peucedanum officinale.

-. - - Silaus.

Laferpitium lntifolium. (Muggend.) Heracleum Spbondyizum.

Angelica fylueftris.

Gentiana.Amnella. (ad mont. pir.) - - Archangelica. (ad fl. Wifendt.)

- $\quad$ - cruciatn. (Fraucnaurach.)

- ciliatn.

Eryngiun campcftie. (Cayerliud.) Sium Falcaria. (Muggendorf.). Oenanthe fiftulofa.

Phcllandrium aquaticum:

Cicuta virofa.

Hydrocotyle vulgaris. (Secbach.) Sanicula europaea.: (Erl. Pegniz.). Bupleurum rotundifolium. (Rathsb.. - - falcatum. (Streitb.Sanspar.) Tordylium Antbrifitus.

Aethufa Cynapium. (Rathsberg.) -- -. Merm. (Warmenfteinach.) Scandix pecten Veneris. (Rathsb.) - Authrifcus. Chacrophyllum fylueftre..

Chacro.

Chenopod: mural: Flores in cymam congefti.. Clienop.ab. Racemi fpicati. Chenopod: virid. Racemi ramofi aphylli tandem.

Chenop. glauc. Racem. Implices.

Peucedanum afficinale inter Illeshein et Burgbernhrion provenire, refert Perill. Def rv s, Fautor ac Praecepfor ad cineres ufque venerandus, in Differt. de Peucedano germanico. Erlang. 175 i. 4.

Aethufa Mevin. Invoiucrum univerfale 1 - 2 phyllum lineare, longitudinis fere radiorum, apice pinnatifidum, deciduum. Involucrum partiale. Foliola linearia 2. 3. 5 phyll. 
Chaerophyllum bulbofum.

- - temlinl:min.

- bivfutwm. (Muggend. Bayr.)

Paftinaca fativa.

Carum Carvi.

Pimpinella Saxifinga.

magna.

Apium graveolens. (Muggendorf.)

Acgopodium Podagraria.

Viburnum Lantana. (Erl. Muggd.)

Opulus.

Sambucus Ebulus.

nigra.

racemofa.

Staphylea pinunta. (Muggendorf.)

Alfine medin.

\section{Tetragynia.}

Parnaffia paluftris. (Erl. Baruth.)

\section{Pentagynia.}

Statice Armeria. (Lilang. etc.)

Linum catbarticum. (Erlang.)

\section{Radiola. (Erlang.)}

Drofera rotundifolia. (Erl.m. pin.) Myofurus minimus. (Uttenr.)

VI. HEXANDR. Monogynia. Levcoium vernum. Allium oleraceum.
Allium nv.jinum. (Muggendorf.) Lilium Murtngin. (Muggendorf.)

Tulipa fyiveftis. (Culmbaci.)

Ornithogalum latcom.

- - minimum. (Erlang.)

Anthericum ramofum. (Muggend.)

- Lilingo. (Oberndorf.)

Afparagus officinalis.

Convallaria majalis.

-.-Polygonatum. (Sanspareil.)

- bifolia. (Erlang.)

Acorus Calnmus.

Iuncus conglonerntus.

- effisus.

- filiformis. (Fichtclb.)

- Squarrofus.

- articulatis.

- bulbofus.

- bufonius.

- campeftris.

Berberis vulgaris.

Peplis Portula.

Digynia.

Rumex Patientin. (Muggendorf.)

- crifpus.

- maritimus. (Erlang.)

- acutus.

- obtufifolius.

- nquaticus.

B 2

Rumex

Incolae agri Muggend. non Tolum baccas Vib. Lantanae (Kautelweidsbeere), fed etiam Roob exinde paratum contra anginam comedunt.

Var. Iunc. campeffris. Iuncoides latifolium alpinum glabrum, panicula fublutea fplendente. Sсн区YCHz, gram. 314. HALL, helv, n. 1329. difinita species. 
Rumex cingl meratus MVRRAY.

Erica vuigaris.

(Nemo-lapathuin EriRH.)

- Acctofa.

- Acertelina.

Daphine Mezcreum. (Pegn. Sansp.)

Stellera Pafirina. (Rathisberg.)

Triglnchin pri fre. (E.lang.)

Colchicum autumnale.

\section{Polygynia.}

Alifina Plautngo aquatica.

VII. HEPT ANDRIA. Monog.

Trientalis curcpnea. (Pegniz. Fan: taifie et in. monte pinif.)

VIII. octandria. Monog.

Oenothera biennis.

Epilobium anguft folium. (Erlang.)

- birfutum. (ibid.) a. B.

- montanum. (Rathsberg.)

- tetragonum.

- paluftrer (Erl.)

Vaccinium Myrtillus. (Erlang.)

- uliginofum. (Fichtelfee.)

-.. Vitis idnea. (Erlang.)

\section{Trigynia.}

Polygonum Biftorta. Weifenftadt)

- - nmpibibium.

- - Hydropiper.

- - Perficaria.

-.- aviculare.

- Convolvulus.

- - dumetorum.

\section{Tetragynia.}

Paris quadrifolia. (Erlang.)

Adoxa Mofibutellina. (Pegniz.)

Elatine Hydropiper. (Erlang.)

IX. ENNEANDRIa. Hexag. Butomus umbellatus.

X. Decandria. Monogynia. Tribulus aquaticus. (Weiffena.) Monotropa Hypopitys. (Erlang.)

- Oxycoccos. (Erl. Fichtelb.) Ledum paluftre. (ad mont. pinif.)

Andro.

Trientalis estropaea. E verticillo foliorum pedunculi 1 - 2. rarifime 3. Variat 5. 6. 8. rariffine oftaminibus cum totidem calycis laciniis.

Varifci radices Biftortae radula comminutas, oryza aut hordeo, aut fagopyro cotto commifcent, iisdemque ut remedio antidyfenterio utuntur.

Ledo paluftri (fub nomine Schabenkraut) incolae Baruth. fupramontan. tineas abigere folent, pofito in vefinienta. Quin nonmuli hoc eodem cum malto et Lupulo cocto, cerevifiam.adulterant, vim huic inebriantem inde conciliantes. 
Andromeda polifolia. (Fichtelb.) Arenaria trinevia.

Arbutus (T'a uvfi. (verfus Lauf.) - - Serpyllifolia.

Pyrcla otundifolia. Raths. Sansp. - - rubia compcftris. (Erlang.

- minor.

- $\quad$ - fecunda. (prope Wunfiedl.)

- - umbeilata.

Petagynia.

- uniflora.

Sedum Tolephiun.

\section{Digynia.}

Chryfofplenium alternifolium.

- oppofitifolinm. (Eremitage.)

Saxifraga gramulata.

- verna. (Pegniz.)

- - tridaćtylites. (Pègniz.)

- - reflexum.

- - album. (Muggendorf.)

- epetrnea. wiblich. (Muggd.)

Scleranthis annuls ] nom. indig.

- - percnnis J Kniebufs.

Gypfophila muralis.

- acre.

- - Sexangulare.

- villofim. (Erlang.)

Oxalis Acetofella

Suponaria officinalis.

Dianthus Armeria. (Erlang.)

- prolifer.

- deltoides.

\section{Trigynia.}

Cuclibalus bacciferus. (Erlang.)

- Beben.

- comiculata. (Rathsberg.) Agroftemma Gitbago.

Lychnis Flos criculi.

- Vifcaria. (Erl. Berr. Baruth.)

Var. fiore aibo. (Leinek.)

- dioica.

Ceraftium vulgatum.

- vifcofinu.

- arvenfo.

- aquaticum.

Spergula arvenfis.

- pentondra.

Silene mutrals.

Stellaria nomorum. (Eremitage.)

- bolofter.

- graminea.

- Dilleniana moEnch.

XI. dodecandria. Monog.

Afarum elropacum.

Portulaca oleracea. (Erlang.).

Lythrum Salicaria.

B 3
Lytbrum

Dianthus Armeria. Canlis faepe 4 - 5 pedes altus.

Foliis Cucubali Behon (fub nomine Kernkraut) paflim olerum loco vefcuntur. 
Lythrum by dopifolia.

Agrimonia cupatoria.

Reieda Luteola.

Euphorbia Peplus.

- exigua acuta. (Rathsb.)

- bclioficopia.

- platypliyllos. (Rathsberg.)

- Estula.

- Cyparijfias.

\section{Dodecagynia.}

Sempervivum tectorum.

\section{ICOS.ANDRIA. Monog.}

Prunins Padns.

- Cevajis.

- avizmm.

- Spinofa.

Crataegus torminalis. (Azelsb..)

- Oxyacantba. (ibid.)

Sorbus aucuparia.

Mespilus Cotozzeafter.

Pyrus communis.

- Malus.

Spiraea falicifolia."Baruth.Culmb.)

- Aruncus. (Muggd. Barutlı.)

- Filipendala. (Baruth.)

- Ulmaria.

\section{Polygynia.}

Rofa eglanteria.

- rubiginna.

- Spinnfiffimso.

- villoga.

- canina.
Rubus idaeus.

- caefius. (Rathsberg.)

- fruticufus.

- Saxatilis. (Pegniz. Sansuar.)

Fragaria vefica.

Potentilla Anferina.

- verna.

- reptans.

- argenter.

Tormentilla erecta.

Geum urbanum. (Rathsb. Vttenr.)

- rivale. (Erlang. Baruth.) .

Comarum paluftre. (Erlaing.)

XIII. polyandr. Monogyn. Actaea Spicata. (Pegniz. Barutl..) Chelidonium majus.

'Papaver Argemone.

- Rbocas.

- dubizm.

Nymphaea aiba.

-- lutca.

Tilia europaea.

Ciftus Hclianthemum. (Vttent.)

Delphinium Confolida.

Aconitum Lycoctonum. (Muggd.)

Aquilegia vulgaris.

Nigella arvenfis. (Rathsberg.)

Polygynia.

Anemone Hcpatica. . (Ieiligenft.)

- - Puifatilla. (Pegniz.)

- fylucfiris. (Muggendorf.)

- nemorofa. 
Anemone ramunculoides. (Muggd.)

Clematis Vitalba. (Muggendorf.)

- Thalictrum minus. Muggd.)

- flavuni.

- aquilcgifolium. (Creufen.)

Adonis acftivalis.

- vernalis.

Ranunculus Flammula.

- Lingua. (Erlang.)

- Ficaria.

- auticomus.

- Seclcratus.

- aconitifolius. (in m. pinif.).

- bulbojus.

- repenss.

- acris.

- arvenfis.

- bederaceus

- aquatilis. $\alpha . \beta . \gamma \cdot \delta$.

Caltha painfris.

XIV. DIDYN.AM. Gymnorp.

Ajuga reptans.

- Pyramidalis.

- B. Genevenfis.

- Chamaepitys schrebrr.

Teucrium Botrys. (c. -Muggend". ad Streitberg, Spekberg, ad.
Sansp. Vid. ill. scrmer, de plantis verticillat. nnilab.

Teucrium Scordium. ad fl. Aifch)

Nepeta Cataria. (Bruk.)

Mentha fylveftris.

-. rotundifolin.

- - aquatica.

- - arvenfis.

Glecoma bederacea.

Lamium. maculatum.

- albunt.

- purpurèum.

- amplexicaule.

Galeopfis Ladanum.

- Tetrabit.

- -. Galéobdolon.

Betonica officinalis.

Stachys fylvatica.

- paluftris.

- - germanica. (Muggd. Bern.)

- recta. (prope Muggend.)

Ballota nigra.

Marrubium vulgave.

Leonurus Cardiaca. (Bayersdorf.)

- Marrubiaftrum. (Erl.).

Clinopodium vulgave.

Origanum vulgare.

Thymus Serpyllum.

Acinos..

Scutellaria:

Clematis Vitalba interne et externe in decocto adhibita;, ulcera fcrophulofa, antiqua Sanavit.

Rientha aquaticn. Stanina in plerisque exemplaribus corolla minora:

In Menth, arøenf. Stamina corollam fuperantia. 
Scutellaria godericulata.

Prunella vulgarir.

- grendiflora polz. (Tennenl.) Rhinanthus Crifta galli.

$V$ ar. $\alpha$, HALL. 3 I3. $\beta .3$ I Euplirafia officinalis.

- - Odontites.

Melampyrum criftntum. (Erlang.)

- - arvenfe.

- - nenorofmu. (Baruth.)

- - Syluaticum.

Jathraea Squnmarin. (Azelsberg.) Pedicularis paluftiis.

- - Solintica.

Antirhinum Elatinc. (Rathsb.)

- - fpurium. (ibid.)

- - avvenfe.

- minus. (ibid.)

- Linaria.

- mius. (Vttenreuth.)

- Orontinus. (Rathsberg.)

Scrophularia nodofa.

- - a ajuatica.

Digitalis ambigua (Eriang.)

Var. corolla alba.

Limólla aquitica. (ibid.)

Orobanthe maior.

XV. TETRADYN. Silieulofac. Myagrum fativum.

- - paniculnâum.

Draba aizoides. (prope Muggd.)

- vernis.
Lepidium ruderale.

Thlafpi arvenfe.

- perfolintum. (hinter Spard.)

- Burfa paftoris.

Cochlearia Coronopus. (Bayersd.) Iberis amana: ( Muggendorf.)

- nudicaulis. (Erlang.)

Alyfum faxatile. (prope Muggd.)

- - incorum.

- - calycinum.

Lunaria rediviva. (am Schönenft.) anina. (Muggendorf.)

Cardamine Impotiens.

- - - pratenfis.

- - - mmara.

Sifymbrium Nafturtium aquaticum.

- - Syrueftie.

- - $\quad$ ampbibium.

- - Soplin.

Eryfimum officinale.

- - baruarea.

- - Allinria.

- - repandum. (Rathsb.)

- - Cbcirantboides.

- - bicracifolium. (Baruth.)

Chciranthus ery/moides. (Muggd.)

Arabis thnitan.t. (Pegniz.)

Turitis glabra.

- - birfutr. (Erlang.)

Braflica orientalis. (Rathsberg.)

- campejtivis.

- alpina. (Fantafie.)

Braftica

In locis ficcioribus folia Scutellar, galericulat. faepius rugofa reperiuntur. 
Sinapis arvenfss. (Rathsberg.) Orobus tuberofus.

- nigra. (Bictsfeld.)

Raphanus Raphanifrum.

XVI. MONADELPH. Triandr.

Geranium cicutarium.

- - pulufire.

- - pratenfe.

- - - robertianum.

- - columbinum.

- - difjectum.

- - - rotundifolium

- - fonguineun. (Pegniz.)

Maiva rotundifolia.

- - fylucftris.

- Alcea. (Erlang.)

\section{DIA DEL PH. HeXandr.}

Fumaria bu!buga.

- - officinalis.

Polygala amara. (Cayerl. Strcitb.)

- - vulgaris. Var. plures.-

- - Chamacbuxus. (Pegniz.)

Spartium fcoparium.

Genilta fagittalis. (Cayerlindacli.)

- tiaćnria. (Rathsberg.)

- germanica. (Baruth.)

Ononis $\int p \operatorname{mof} a$.

- $\quad$ arvenjis.

Anthyllis Vulneraria.

Orobus veruis.
Lathyrus tuberafus.

- - praterifis.

- - Sylueftivis.

Vicia dumetorum. (Muggd. Sansp.)

- cafjubica. (Erlang.)

- Cracea.

- Sativa.

- fepinm.

Ervum tetrapermum. (Baruth.)

- birfutum.

Cytifus nigricans. (Erl. Baruth.)

Coronilla varia.

Aftragalus glycyphyllus. (Rathsb.)

Trifolium Melilctus officinaits.

- bjbridum.

- - repens.

- mbens. (Neur. ad Aifch.)

- pratenfe.

- Acxucfum JacQ. (Rathsb.)

- ocincleucum. (Erlang.)

$\therefore \quad$ - arvenje.

- - montanum.

- agrarium.

- Spadiceum. (Erl. Sophicub.)

- procumboris.

- fliforme.

Lotus corniculatis.

Merlicago fativa.

- - falcata.

- - lupuliris.

XVIII, no.

Polygalae amarae in phthif pituitofa vires falutares experti fumins. 
XV!II. noN DELPH.Pentand. Hieracium fabaudum.

Hypericum quadrangmlum.

- - dubirm lesers.

- - perforatim.

- - humfufum. (Eriang.)

- - montanum. (Ratlisb.)

- $\quad$ - bivfutumi.

XIX. SYNGENESIA

Polygam. aequal.

Tragopugon pretenfe.

Scorzonera bumilis.

Souchals ainengis. (Rathsberg.)

Lasiuca Seariola.

- percnuis. (Muggend.)

Chondrilla innera. (Eilang;)

Prenanthes purpurca. (Pcgniz.)

- - - simuralis.

Leontodon Taraxacum.

- - - bajtile.

- - bifpillim.

- - - livtum.

Hicraciun? incontm. (pr. Muggd.)

- Pilorclia.

- - d dibiam.

- - Auritia.

- - pracherfino. (Muggh.)

- - - mavinits.

- palutofum. (adm.pin.)
- - - mubilatim.

Crepis tect ormm.

- - bicninis.

Hyoferis minima. (Rathsberg.) Hypochaeris maculata. (Baruth.)

- - glabra. (ibid.)

- - radicata. (Streitberg)

Lapfana commutris.

Cichorium . Intybus.

Aretiun Lappa.

Serratula tinctoria. (Rathsberg.)

- arvenfis.

Carduus lanccolatus.

- mutans.

- $\quad$ acantboidcs.

- Var. Al. albo. (pr. Erl.)

- $\quad$ crifpus.

- palifiris.

- - defloratus. (Muggend.)

- criopionous. (Bretsfold.)

- $\quad$ acoulis.

Cnicus oloraceus. (Rahtsberg.)

Onopordon Acnntbiun

Carina unigaris. (Erlang.)

Bidens tripartita.

- minima. (prope Evlang.)

- - ceraria.

Eupatoriun somabinm.

Varict. Serratmac indorice foliis fuperioribus integris; inferioribus farum piniatifitis.

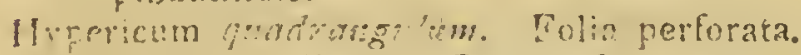

Lypochaejis rodicata. Calycis fquanae carina is çuisusdam exemplaribus Hon cilizta el?. 


\section{Polygamia fupcifua.}

Tanacetum vu!gare.

Artemilia compgeris.

- Albsitbitur. (ad Streitb.)

- vulgaris.

Gnaphalium arinarium.

- luteo-alumm. (Evlang.)

- - dioicum.

- stiginofum.

- Jilvaticum.

Conyza fijuarrofa.

Erigeron canadenfe.

- - acre:

Tuffilago Favfara.

- bybrida. (Erlang.)

Senccio vuigaris.

- vifiofus.

- Sylunticus.

- crucifolius.

- - Incobaen.

- paludofus. nenorenfis. (ad $\mathrm{m}$. pinif.)

Solidago Virga aurca.

Inula Eritannica.

- dysenterica.

- Pulicaria.

- falicina. (Soplicuberg.)

Amica montana. (Bar. Mcencli.)

Bellis percruis.

Chryianthemum Leucantbomim.

- corymbiferum (Muggod.)

- Segetim.

Matricaria Chamomilla,
Anthemis arverytis.

- - Cotula.

- - tinctoria.

Achillea Ptarmica.

- - Millefolizm.

Buphthalnum falicifolium.

Polygamia fruftranca.

Coreoplis Bidens.

Centaurea plorygin. (ad m. pinif.)

- - Cynnus.

- - Scabiofa.

- - Incea.

\section{Polygamia neceffaria.}

Filagó germanica.

- Montara. (Rathsb.)

- arvenfis.

\section{Monogamia.}

Iafione montana.

Vioia birta.

- prilufiris.

- - odorntro.

- cninina.

- tricolor.

Impatiens Noli tangcre.

X.. GNandra. Diandria.

Orchis bifolia. (Murgenendorf.)

- coriopluora. (Sanspareil.)

- morio.

- mafiulas

$\mathrm{C}_{2}$

Orchis 
Oichis uftulatu.

- militaris. Muggd. Baruth.)

- latifolia. (Mugrendorf.)

- maculata.

- conopfera.

Satyrium lircinum.

- - vivide.

Ophrys Nidus avis.

- Spiralis.

- - ovata. (Barnth.)

- infectifera. (Muggendorr.)

Serapias latifolir (ibid.)

- grandiflora. (Drofienf.)

- rubra. (Muggendorf.)

Cypripedium Calceolus.

(Hochitatt, Spies, Blech.)

Arilfolocinia Clematitis.

Arum suaculatum.

Calla paluftris.

XII. Monoecia. Monandria.

Chara ftexilis. (Bukeninof.)

Lannichellia paluftris. (Err. Barth.)

I.cmna trifulia.

- minor.

- sibba.

- poiyrrbiza.

Typha latifolia.

Sparganium erećtum.

Carex disica.

- difficha huvonon. (Pollich.)

- leporina.

- vulpina.

- brizoides. (Erlang. Pegniz.)
Carex remota.

- elongata.

- cancfins.

- pariculata.

- diandra schrask.

- Alava.

- digitata.

- montana. (Pegniz.)

- praccox IAcro,anftr.t.446.

- tomentofi.

- pallefiens.

- panicea.

-. Peudocyperus.

- cacjpitofa.

- diftans.

- acuta nigra.

- - - rufa.

- Spadicea scinevchz. 465.

- flacer schr. (glauc. poll.)

(Rathsb. Muggd. Pegn. ad m. pin.)

- bivta.

- Syluatica HVDSON.

- Spicat. HVDs. interm. RETz. Betula alba.

- Alnus.

Vrtica ureus.

- dioica.

Xanthiun frumarium.

Ainaranthus Blitun.

Polyandria.

Ceratophyllum demerfum.

Myriophyllum Spicatum.

- - . verticillaturs. 
Sagittaria fagittifolis.

Poterium Sanguiforba.

Quercus Robur.

Fagus Syluatica.

Carpinus Betulus.

Corylus Avellana.

Pinus fylveftris.

\section{- Picea.}

Bryónia alba.

\section{DIOECIA, Diandria.}

Salix triandra.

- pentandra.

- ploylicifolia: (prope Pegniz.)

- vitellina.

- amygdalina.

- fragilis.

- monandrntiofr. (Erlang.)

- fiffa nоғтм. (prope Erlang.)

- aurita.

- depregia horfm. (Tennenl.)

- caprea.

- aclininata MILLER.

- viminalis. Var. A. B.

- mollifjimn Horrm. (Erlang.)
Viscum albrtm.

Humulus Lipulus.

Popnlus alba.

- - tremuila.

- nigia. (Baruth.)

Iuniperus conmmnis.

Taxus baciatn. (Muggendouf.)

\section{POly gam. Monoec.}

Holcils mollis. 
Ophiogiofrum trigatunt.

Osmunda Lusnavia.

- - fuicant. (in m. pinif.)

Acroftichum Septentrionale.

Pteris iquilina.

- - Tricbosianes.

- - Ruta miviravia.

Polypodium criftatum. (in m.pin.)

- - vulgare."

- Plsegopteris.

- Fiix mas.

- - - fcmina.

- - aculcntum.

- - molle.

-. fringile.

- - Dryopteris.

Pilularia globulifora. (Erlang.)

Lycopodium clavatum.

- inunántum.

- Selngo.

- complanatum. (Bulienh.)

Sphagnum paluftie.

Phafcum nemule.

$\therefore$ - Jubulatuin.

Fontinalis antipyretica.

\section{- ., minor.}

Buxbaumia apbylla.

Splachinm ampullaceuri. (m.pin.)

Polytrichum commune.

Muium pelilucidum.

- - fontinumo.

- palufire.

- bygromstricurs.
Mnium pupurcum.

- - fetaceam.

- cirratum.

- annotinum.

- bornitin.

- crudun.

- polytrictsoides.

- Scrpyllifolinancufpidatum\&e.

- triquetrim.

- Triclomanis.

Bryum spocaspum.

- friatum.

- - pomiforme.

- pyriforme.

- extinctorium.

- Sibulatuin.

- - rurale.

- minrale.

- Scopariunn.

- undulatum.

!' glauculi. .

- poliucidum.

- - unguiculatum.

- betcroainallim.

- truncntulum.

- - viridulun.

- byproiles.

- trichodes.

- - argenterm.

- pulvinatum.

- crofpititian.

- capillave.

- fimplex.

Afplenium Srolopendrium circa Efclenbach provenire, aicitur. 
Iypnum taxifolium. denticulatum. bryoides. adiantoides. complanatum. rugofum. undulatum. crispur\%. triauctrum. vitabulum. flicinmm. prolifernm. parictinum. praelongutur. Crifta caftrenjis. al:cinizim. pluinofinn. cuprelliforme: nduncum. Squarrofim. dendroides. alopecurum. curtipendnlum.

- privum.

- cuspidatum.

- - ripariam.

- Sericum.

- - vclutinum.

- Serpons.

- Sciuroides.

- myofriroilics.

Iungermannia afpienioides.

bidentata. bicufinitatu.
Iungcrmannia quinquedentata.

- - refupinatr.

- complaniatx.

- dilatata.

- - tamarifcifoliz.

- platypisylla.

- ciliaris.

- rupcftris.

- tricbopisyila.

$\therefore$ pinguis.

- - furcata.

- pufilla.

- ferpyllifolia.

Marchantia polymorpha.

- conica.

- androgjina.

Blafia prtilla. (Erlang.)

Ricia cryftallina.

- minima.

- glancr.

- natans.

Authoccros punctatus.

Ord. I. Licbenes pilverulenti. Lepra.

Licicnantiquitatis LINN. (Streitb.)

- flavus SCHREBER.

- lutefiens HOFFAIANN.

- rubens IIOFFMANN.

- - rofcus scirreser.

- botryoides rinN.

- laztcus schrtier (no pin.)

- - incenus sCHRexler. 
Lichen farinofis HOF FMANN.

- tuberculofis hof EMANN.

- ferrugineiis hoffanan.

Ord.II. Licben. leprofitubercul:

\section{Verrucaria.}

*) Tuberculis cavis, pulvere repletis. Lichen rugofits LINN.

- firiptus LiNN.

- hebreicus lightfoот.

(Var. a. Lich. Seript.)

- pulicaris lightfoot.

(Var. B. Lich. feript.)

- - pertufus LTNN.

- figineus LxNo.

**) Tuberculis folidis.

Lichen cindidus nofFM. (lact.LINN.

- - riaticola LINN.

- immerflus weder. (Streitb.)

- rapefris scopol. (Streitb.)

- graniformis. HAGEN.

- Sangenizarius LINN.

- albo-ater hoffanan.

- cucruleo - nigricans lightr.

- calcarinls LiNN.

- mufcortun wereir.

- - filfurecis nof fin. (m. pinif.)

- polynorplus hoffar. (Pegin.) **) Tuverculis feflilibus fung ofis.

Lichen vernalis LINN.

-: icmadoplila. enrinart.

- aeruginofus scopol.
Ord. III. Lichenes lebrofi tuberculati fungof ftipitati.

\section{Tubercularia.}

Lich en fabulctorum scHREBER.

- breomyces ehrhart.

- fungiformis scopol. (Raths.)

- decipiens eirriakt.

Ord.IV. Licb.leprofifcutcliati. Scutellaria.

Lichen corallinis LINN. (m. pin.)

- Scrupofus schreb. (Muggd.)

- alzantiacus lightroot.

- caerulcfenis HAGEN.

- cxcruatus hoffarans.

- fulvuis schreser.

- carpineus LinN.

- angulofus schreder.

- pallidus norfanas.

- fubfufcus LINN.

- ater hivdson.

- parellas linNo (m. pinifo)

Ord. V. Licb. cruftac. imbric. Piora.

Lichen candelarius LINN.

- fluveficus hvoson.

- lendigerus web. (Muggd.)

- candidtls werer.

- miniatus Hofra. (Strcitb.) 
Tichen muraran HOEFM.

(Sricola pollich.)

- meralis sCHREBER.

- cacfiźs horfa.

- gramizus schreb. (Pegno)

- pailefecils necler.

- orbiculavis hofFm.

Ord.VI. Lich. imbricatifoliac.

\section{Lichen.}

*) foliolis imbricatis linearibus.

Lichen ciliatus HofFM.

- diffulus hoffm.

- fallaris LinN.

- fellariformis Hofrm.

- fufcus hivds. (m. pin.)

- pulverulentus schreb.

- angufratus horFM.

**) foliolis imbricatis latioribus.

Lichen centrifugus LINN.

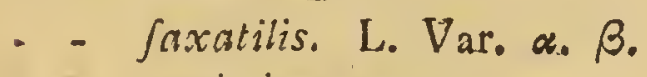

- parictinus linN.

- caperatus LiNN.

- olizacms LINN.

- tiliaceus hoffm.

- glaucus LINN.

***) Solids imbricatis inflatis.

Lichen pliyfodes LINN.

- tenellus schreber.

- bijpidus scopol.

Ord. VII. Tremellofi.

Colloma.

Lichen crifpus LINN.

- criftatus LINN.

- trentelloides Leers.

- aigrefents rivds.

(vefpertilio LIGHTF.)

Ord. VIII. Lichenoides.

Foliis ereetis.

Licheì islandicus LINN. (Erlang.)

- furfuracens LINN.

- farinaceus LINN.

- fraxineus LINN.

- pruniaftri LINN.

- ciliaris LINN.

- fallax wer. Linn. (m.pin.)

Coriacei.

- pulmonaritis LINN.

- refupinatus LiNN.

- polydactylon wEBER. (ErT.)

- - mifefiens werer.

- apbthofus LinN.

- borizontalis LIVN.

- caninus linv. B amplifim.

- faccatus lin. (Pegniz.)

- complicatus Ligirt.(Strcitb.

$\checkmark$ imbilicati.

- - miniatus LINN. (Pegniz.)

- velletts LINN (m. pinifer.)

Lichen

Lichen isiandicus in phthifi ulcerofa nec nen pituitofa, ac febre proniganda, vim fuam exferuit. 
Jichen prefulatus LINN.(ad Steitb.) Lichen floridus urNw.

- trapeaifurmis retz. ibid.) - - lanatus LINN.

- - micropbyllhs ehrhart.

Tremella Noftoc.

\section{Scyphiferi LINN.}

Lichen cocciferus

- pyxicintus.

- fimbrita.

- gracilis.

- digitatus LINN.,

- deformis LINN.

- fquamofus scopol.

- folincels schreber.

Fiuticulofi LINN.

Richen uncialis LINN.

- ramgiferinus LINN.

- pafchalis LINN. (Erlang.)

- fragilis LINN.

(il.. pinif.)

- aculeatus scirreber.

- ceranoides necker.

- cafancus leers (Muggd.)

- Jpinofirs nuvos.

- bypotrichodes weEER.

Filamentofi IINN.

Iichen plicatus LINN.

- baibatus LINN.

- duaricatus LinN.

- jubatus LIXN. -

- cisalybeiformis uniN.

- birtus isinN.

- $\quad$ mefenteriformiso

Conferva rivilaris.

- ballofa.

- ampliblin.

- reticulata.

- fuviatilis.

- capillaris.

- glonerata.

Byffus Septica.

- Flos aquar.

- - plofpisorea.

- velutina.

- aurea.

- botryoides.

- incana.

- laçter.

- Penigillum scor.pI. fubterr.

Agaricus cantbarellis.

- integer.

- mufenvius.

- deliciojiss.

- lnctifiuus.

- pipcratus.

- compeftris.

- cincreus schaEfEET.

- cinamometis.

- vio?accus.

- Georgii.

- finstarius

Aggarius

Lichen pyxidatus, in tuff ferina fumto aniea emetico, efficxiam fuam nuper praeftitit. 
Agratcus minceus.

- adnajcens.

- qucrcinus.

- betulinuss.

- jejpticus. bvlliard.

- fterconaritls BVILIARD.

- nlnezs.

- niveus scovoL.

Boletus fuberofus.

- igniarius.

- cinnabarimis.

- verfucolor.

- funveolens.

- Medulla panis.

- luteus.

- bovinus.

- - coriaceus SCHAEFEER.

IIyanum imbricatum.

- - repandum.

- tomentofujo

- aurifcalpium.

- - parafiticum.

Phallus efculczitus.

- imprudicus. (Erlang.)

Clathrus cancellatus.

peziza lentifera.

- prenctata.

$\therefore$ - corthucopioides.
Peziza acetabuluss.

- cyatboides.

- - cupularis..

- fiutcllata.

- Auricula.

Clavaria caefpitoja.

- - opisiogioffoides.

- - digitata.

- - bypoxylon..

- - corntioides.

- mufcoides.

- teres fiftul. etc. scrinmér.

- - fimplex ctc. schmidel.

Eycoperdon Tuber.

- cervinums.

- minimum.

- Boviftro

- ftcllatum.

- Carpobolus.

- - Sanguineum. (Fichtelib.)

- voluam recollig. Schnid.

- variolofim.

Mucor Splacrocepbalus.

- Embolus.

- furfuraceus.

- - rufus lEERS.

- Muccdo.

- glaucus.

- cruftacèus.

(Monilia cruftacer wrgats.)

$\mathrm{D}_{2}$

Mucor

Iucolae Baruth. fupramontani Agaricum alliabrim concifum indere folent in farcimina,

Boletus fuaveolens faepins in phthifi, ex humorum acrimonia exorta, fuam vim nobis iterum comprobavit. 
Mlucor fep: iels.

Triclia cociner. HALT. 2164.

- pyiformis HAtc. 2167.

Splaeriatingiformis HALL.

- deufta Michel. (Streitb.)
Merulius pedatus. (im Stebn. Stoll.

Poria pulvinata scop. pl. fubtert. Fungus Chirothecn scop. pl. fubt. (im Stollen zu Steven.)

Nerulitis pedalus feffilis coriaceus aurantiacus, fubtus fulvus, venofe plicatus. Lo'stgitud. faepius 15 . pollic.

Fungus Chrobheca. Lamina tenuis, bafi attenuata, apice laciniata.

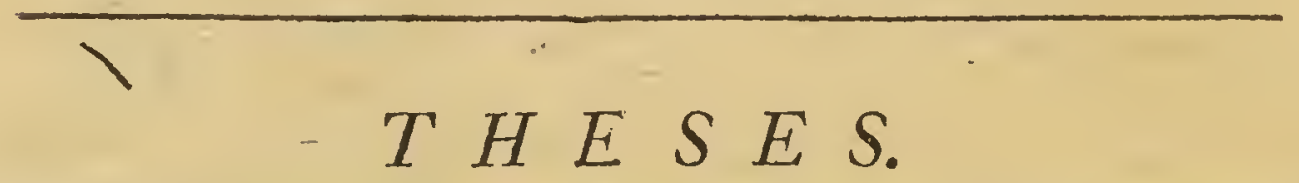

I. Matris imaginatio plurimum valet ad formationem et deformationcm foetus.

2. Equitatio in phthif mortem promovet.

3. Morbis melancholicis fine adhibitis clyfinatibus mederi non porfumus.

4. Olca coeta ex officinis pharmaccvticis reiicienda funt.

5. Aqua plagednenica non eft corrofiva.

6. Hydralgyrum nihil valct contra tacniam.

7. Fructus Pruri Cereolac (Spillinge), in dyfenteria potius profunt, quam nocent.

8. Ommibus medicamentis exoticis facile carcre pofumus.

9. Inter caufas morbificas ipfe foetus referendus cft.

ro. Confect. Alkerm. et de Hyacintho cx ofticin, pharmac. relegandae fint.

II. Succinum pracparatum interne fumtum vi medica deftitutum eft.

I2. Oleum ex amygdalis amaris cxpreffum, amarum non fit.

I3. Cryftallus montana pracparata, intus afuntä venenum crt. 







